\title{
Modality exclusivity norms for 423 object properties
}

\author{
DERMOT LyNOTT AND LOUISE CONNELL \\ University of Manchester, Manchester, England
}

\begin{abstract}
Recent work has shown that people routinely use perceptual information during language comprehension and conceptual processing, from single-word recognition to modality-switching costs in property verification. In investigating such links between perceptual and conceptual representations, the use of modality-specific stimuli plays a central role. To aid researchers working in this area, we provide a set of norms for 423 adjectives, each describing an object property, with mean ratings of how strongly that property is experienced through each of five perceptual modalities (visual, haptic, auditory, olfactory, and gustatory). The data set also contains estimates of modality exclusivity - that is, a measure of the extent to which a particular property may be considered unimodal (i.e., perceived through one sense alone). Although there already exists a number of sets of word and object norms, we provide the first set to categorize words describing object properties along the dimensions of the five perceptual modalities. We hope that the norms will be of use to researchers working at the interface between linguistic, conceptual, and perceptual systems. The modality exclusivity norms may be downloaded as supplemental materials for this article from brm.psychonomic-journals.org/ content/supplemental.
\end{abstract}

Concept properties, also termed object attributes or semantic features, have long been key to understanding a wide variety of cognitive phenomena from categorization (Medin \& Schaffer, 1978; Osherson \& Smith, 1981; Rosch \& Mervis, 1975; Sloman \& Malt, 2003) to semantic memory deficits (Martin, 2007; Tyler \& Moss, 2001; Warrington \& Shallice, 1984), and assumptions of how concepts and properties are represented also underlie competing theories in other areas, such as discourse comprehension (e.g., Kintsch, 2001; Zwaan, 2004) and infant word learning (e.g., Cimpian \& Markman, 2008; Smith $\&$ Samuelson, 2006). Recent years have seen a confluence of opinion that conceptual representation cannot be successfully studied by marginalizing the roles of body, world, and action, while, at the same time, much empirical research has highlighted the relevance of perceptual modalities in conceptual thought.

\section{Modality-Specific Conceptual Representations}

In both behavioral and cognitive neuroscience research, although fine-grained sensory distinctions have long been noted, more recent work has highlighted the continuity between conceptual and perceptual knowledge with respect to the different sensory modalities. Such findings relate to embodied theories of cognition, which argue that conceptual thought is grounded in the same neural systems that govern sensation, perception, and motor control (Barsalou, 1999, 2008; Glenberg \& Kaschak, 2002; JohnsonLaird, 1983; Pecher \& Zwaan, 2005). For example,
González et al. (2006) found that passively reading scentrelated words (e.g., cinnamon) increased activation in the primary olfactory areas of the piriform cortex (similar to Pulvermüller's, 2005, finding of motor cortex activation for action words). Regarding visual processing, Simmons et al. (2007) showed that verifying color properties in text (e.g., that a banana is yellow) led to activation in the same region of the left fusiform gyrus in the visual cortex as that activated by a perceptual task that involved judging color sequences. Similarly, Newman, Klatzky, Lederman, and Just (2005) examined visual and haptic modalities by asking participants to compare various objects and found differential activation in the inferior extrastriate and intraparietal sulcus, depending on whether visual features (e.g., which is bigger? pear or egg) or haptic features (e.g., which is harder? potato or mushroom) formed the basis for comparison. Further comparisons by Goldberg, Perfetti, and Schneider (2006) showed that verification of color, sound, touch, and taste properties activated cortical regions associated with encoding visual, auditory, haptic, and gustatory experiences, respectively, illustrating that perceptual experience and conceptual knowledge share a common neural substrate.

Other recent work has focused on the emergence of perceptual phenomena, such as modality-switching costs, in conceptual processing (e.g., Marques, 2006; Pecher, Zeelenberg, \& Barsalou, 2003). For example, Spence, Nicholls, and Driver (2001; see also Turatto, Benso, Galfano, \& Umiltà, 2002) asked people to indicate the left/ 
right location of a series of perceptual stimuli and found that switching modalities from one trial to the next (e.g., from a visual light flash to an auditory tone) incurred a processing cost. Pecher et al. replicated this paradigm in a conceptual task by asking people to verify a series of object properties presented on-screen as text and found that people were slower to verify a property in a given modality (e.g., auditory, leaves: rustling) after verifying a property in a different modality (e.g., visual, apple: shiny) than after verifying a property in the same modality (e.g., auditory, blender: loud). Like the modalityswitching costs found by Spence et al. during perceptual tasks, such costs during conceptual tasks result from the reallocation of attention from one modality-specific system to another.

However, it is overly simplistic to assume that object properties are conceptually processed only in single, modality-specific regions of the brain. From philosopher John Locke (1690/1975) to modern empirical studies (Amedi, von Kriegstein, van Atteveldt, Beauchamp, \& Naumer, 2005; Connell, 2007; Ernst \& Bülthoff, 2004), many have pointed out that object properties exist in both multimodal (perceptible by multiple senses) and unimodal (perceptible by only one of our senses) forms. For example, whereas the color yellow is normally perceived only through the single modality of vision (i.e., color is a unimodal property), the property round would be considered multimodal, since it can be perceived both haptically and visually. Such distinctions are extremely important when considering interactions between linguistic, conceptual, and perceptual systems (see, e.g., Connell, 2007) and yet, to date, have been largely neglected in work examining modality-specific activations in conceptual processing. A common assumption in empirical studies has been that each object property or word can be mapped to just one perceptual modality (e.g., González et al., 2006; Marques, 2006; Newman et al., 2005; Pecher et al., 2003). For example, Pecher et al.'s classification of rustling as an auditory property may be problematic if the rustling movement of leaves can also be visually perceived and represented. Similarly, Newman et al.'s treatment of rough as a purely haptic feature may introduce unwanted noise, since it is also possible to gauge surface texture by sight (e.g., we can tell that a cactus is prickly or that an egg is smooth without recourse to haptic interaction). Indeed, Newman and colleagues noted that the intraparietal sulcus, a region usually involved in visual imagery, was found to be highly activated when participants were processing the roughness of objects. In short, since many object properties appear to be perceptible through a number of modalities, it is essential for researchers seeking robust experimental designs to avoid assuming unimodal representations for multimodal properties.

\section{Purpose of the Norms}

Our goal in the present article is to present a set of norms for a collection of possible concept properties that measures (1) the degree to which each property can be perceived through auditory, gustatory, haptic, olfactory, and visual modalities and (2) the modality exclusivity of each property (i.e., the degree to which it can be mapped to a single perceptual modality). Existing sets of norms that include modality-specific information in their description of semantic features (McRae, Cree, Seidenberg, \& McNorgan, 2005; Vinson \& Vigliocco, 2008) tend to involve the assumption that an individual feature corresponds to a single perceptual modality. Our empirically derived measures enable us to calculate an exclusivity score that allows each object property to be classified as unimodal, bimodal, or multimodal, while separately considering the representational strength on each perceptual modality. Such information on modality exclusivity can be used in conjunction with existing large-scale semantic feature norms (e.g., McRae et al., 2005) and psycholinguistic databases such as the MRC (Wilson, 1988) and the English Lexicon Project (Balota et al., 2007). In experiments in which modality-specific conceptual representations are of interest, the norms presented here will help to reduce noise in stimulus sets and will allow more subtle main effects and interactions to be detected and explored. By making these norms available, we hope that they will prove useful to researchers interested in testing theories of language comprehension, mental representation, and the interactions between our perceptual and conceptual systems.

\section{MODALITY NORMING STUDY}

\section{Method}

Participants. Fifty-five native speakers of English participated in the experiment for course credit or a fee of $£ 5$. The participants were recruited via university e-mail lists and notice boards and had no reported sensory or reading disabilities. The data from 1 participant who failed to complete the task correctly were removed prior to analysis.

Materials. A set of 423 words was collected from a range of sources (dictionaries, thesauruses, etc.), where each word described an object property that could pertain to one or more sensory modalities (e.g., crackling, glowing, thin, acidic, yellow). All of the words were prenominal adjectives (i.e., could immediately precede the noun, as in yellow banana). Although the properties were not classified a priori, our aim was to generate a wide range of adjectives covering all five perceptual modalities. This set was then randomly split into three lists of 141 items, which were presented to the participants for rating. The complete set of properties is available in the archived file (see the Appendix for variables).

Procedure. Properties were presented randomly to the participants, using Slide Generator software (Tucker, 2007). One property was presented per screen, with a line that read, "To what extent do you experience something being WORD" (with the WORD slot filled by the property in question). Underneath this line were five separate rating scales for each perceptual modality, labeled "by feeling through touch," "by hearing," "by seeing," "by smelling," and "by tasting." The participants were asked to click on the scales to rate the extent to which they would experience each property through each of the five senses, from 0 (not at all) to 5 (greatly). Once the property had been rated on all five modalities, the participants clicked on the "Next" button to advance to the subsequent item. The participants were told that there were no right or wrong 
Table 1

Mean Strength Ratings (0-5), Standard Deviations, and Standard Errors per Perceptual Modality

\begin{tabular}{lccc}
\hline Modality & $M$ & $S D$ & $S E$ \\
\hline Visual & 3.57 & 1.16 & 0.06 \\
Haptic & 2.15 & 1.60 & 0.08 \\
Auditory & 1.48 & 1.60 & 0.06 \\
Olfactory & 1.25 & 1.49 & 0.07 \\
Gustatory & 1.32 & 1.59 & 0.08 \\
\hline
\end{tabular}

answers and that they should use their own judgment; they were also told that if they did not know the meaning of the word, they should skip it by clicking "Next" straightaway to move on to the following item. The experiment was self-paced and lasted from 45 to $60 \mathrm{~min}$.

\section{Results and Discussion}

The participants' responses were collated, and mean ratings per modality were calculated for each property. Thus, each property could now be represented by a fivevalue vector reflecting that item's experiential strength on each modality. Most participants were familiar with the meaning of most of the words $(M=95.5 \%)$. We include these familiarity scores for each item in the data set, representing the proportion of participants who believed that they knew the word's meaning.

In Table 1, we provide the means, standard deviations, and standard errors of the means of the ratings for each of the five modalities. Each property was assigned a dominant modality (visual, haptic, auditory, olfactory, or gustatory) according to its strongest perceptual modality (i.e., the one that received the highest mean rating). Where ties existed for the strongest modality (4 properties out of 423), one tied modality was chosen at random for the dominant label. Table 2 shows the distribution of items and ratings for each dominant modality.
Relationship between the modalities. Not all the perceptual modalities were equally distinct, as is shown in Table 3's correlation matrix for ratings on the five modalities and Figure 1's scatterplot of modality clusters. In Figure 1, ratings on the five modalities have been reduced to two factors, using principal components analysis (unrotated solution, explaining $74.8 \%$ of the original variance). There were significant correlations for the majority of the modality pairs, although most of them were negative and weak to moderate. Indeed, auditory ratings correlated negatively with all the other modalities, which, together with its distinct cluster in Figure 1, suggests that auditory experience has little in common with other types of perceptual experience. The strongest positive relationship was between the olfactory and gustatory modalities (as shown by their clustering in Figure 1), which is perhaps unsurprising given the close association between the taste and smell of foodstuffs. To a lesser extent, a positive relationship also appeared in the visual-haptic cluster, indicating that many properties experienced through touch can also be seen. Only gustatory and haptic ratings showed no appreciable relationship.

Modality exclusivity. Modality exclusivity is a measure of the extent to which a particular property is perceived through a single perceptual modality. Where each property has a vector containing mean strength ratings for all modalities, modality exclusivity is calculated as the range of values divided by the sum. Thus, modality exclusivity scores range from 0 to 1 (i.e., $0 \%$ to $100 \%$ ), where an entirely multimodal property (scoring equally strongly on all perceptual modalities) will have the lowest modality exclusivity score of $0 \%$ and an entirely unimodal property (scoring zero on all but one perceptual modality) will have the highest modality exclusivity score of $100 \%$. Modality exclusivity scores in the norms ranged from $9.6 \%$ to $98 \%$, with an overall mean of $46.1 \%(S D=16.9 \%$; see Table 2 for

Table 2

Number of Properties per Dominant Modality, With Mean Strength Ratings (0-5) on the Five Perceptual Modalities and Modality Exclusivity Scores

\begin{tabular}{lccccccc}
\hline \multirow{2}{*}{$\begin{array}{l}\text { Dominant } \\
\text { Modality }\end{array}$} & \multicolumn{3}{c}{ Strength } & \multicolumn{2}{c}{$\begin{array}{c}\text { Modality } \\
\text { Exclusivity }\end{array}$} & $N$ \\
\cline { 2 - 5 } & Visual & Haptic & Auditory & Olfactory & Gustatory & Excry \\
\hline Visual & 4.4 & 2.3 & 1.0 & 0.8 & 0.7 & $49 \%$ & 205 \\
Haptic & 3.4 & 4.3 & 1.1 & 0.7 & 1.3 & $37 \%$ & 70 \\
Auditory & 2.3 & 0.7 & 4.6 & 0.2 & 0.2 & $57 \%$ & 68 \\
Olfactory & 2.2 & 0.8 & 0.4 & 4.5 & 2.5 & $43 \%$ & 25 \\
Gustatory & 2.8 & 1.1 & 0.4 & 3.3 & 4.5 & $35 \%$ & 55 \\
\hline
\end{tabular}

Table 3

Correlation Matrix for Mean Strength Ratings per Item on the Five Perceptual Modalities

\begin{tabular}{lccccc}
\hline & & \multicolumn{4}{c}{ Modality } \\
\cline { 2 - 6 } Modality & Visual & Haptic & Auditory & Olfactory & Gustatory \\
\hline Visual & 1 & $.378^{* *}$ & $-.340^{* *}$ & $-.269^{* *}$ & $-.249^{* *}$ \\
Haptic & & 1 & $-.238^{* *}$ & $-.233^{* *}$ & -.086 \\
Auditory & & 1 & $-.360^{* *}$ & $-.346^{* *}$ \\
Olfactory & & & 1 & $.782^{* *}$ \\
Gustatory & & & & 1 \\
$*^{* *} p<.001$ (two-tailed). & & & &
\end{tabular}




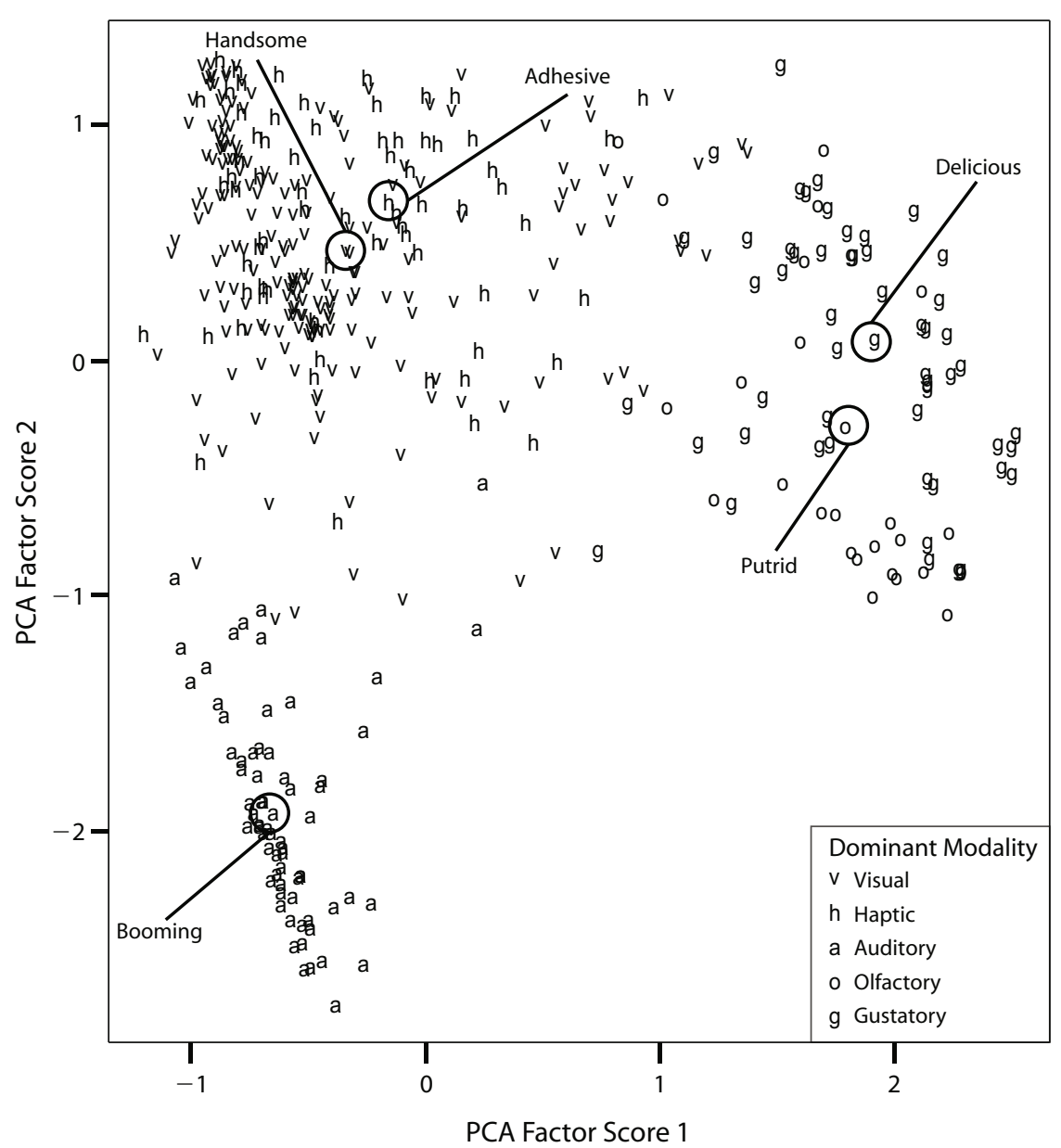

Figure 1. Scatterplot of relationship between properties, labeled by dominant modality, with relative strength on five perceptual modalities reduced to two dimensions by principal components analysis (PCA).

means per dominant modality). Exclusivity scores differed across dominant modalities $[F(4,418)=25.06, p<.001]$, reflecting the clustering shown in Figure 1. Comparisons with Bonferroni corrections showed that properties with auditory dominance scored higher in modality exclusivity than did those for all the other perceptual modalities (all $p$ s $<.01)$. Properties that were predominantly visual were the next most exclusive, more so than haptic and gustatory properties $(p \mathrm{~s}<.001)$ but equivalent to olfactory properties $(p>.7)$, which were in turn more exclusive than gustatory properties $(p \mathrm{~s}<.05)$, but not haptic properties $(p>.4)$. Modality exclusivity was equally low for properties with gustatory and haptic dominance $(p>.99)$.

Since the collected ratings indicate how strongly a property is perceived through a particular modality, they can also be used in conjunction with the modality exclusivity score to generate fine-grained distinctions in experimental materials. For example, if the experimenter requires a set of unimodal object properties, only items with a high modality exclusivity score should be chosen (e.g., $65 \%$ or higher), leaving the corresponding strength on the dominant modality to be similarly restricted or treated as a covariate. On the other hand, if an experimenter wants to concentrate on bimodal visual-haptic properties, items could be limited to only strongly visual-haptic words (e.g., a rating 3.5 or higher on both modalities), where, this time, the modality exclusivity score could be restricted or treated as a covariate. We suggest that a reasonable means of categorizing modality strength (when each modality is quantified separately on a $0-5$ ratings scale) would be $0-1.5$ (weak), 1.5-3.5 (moderate), and 3.5-5 (strong). Similarly, modality exclusivity scores could be categorized as $0 \%-35 \%$ (low), $35 \%-65 \%$ (moderate), and $65 \%-100 \%$ (high). Table 4 gives a sample of items from the data set that have been characterized according to these guidelines.

\section{EVALUATION OF THE NORMS}

In order to test our claim that these norms will allow researchers to detect more subtle modality-specific effects during conceptual processing, we ran a replication of Pecher et al.'s (2003) modality-switching cost experiment. Pecher et al. found that people incurred a processing cost when switching attention from one modality to another (e.g., from auditory leaves: rustling to visual 
Table 4

Examples of Items From the Main Data Set, Along With Mean Strength Ratings (0-5) for the Five Modalities and Modality Exclusivity Scores

\begin{tabular}{|c|c|c|c|c|c|c|c|}
\hline \multirow[b]{2}{*}{ Property } & \multicolumn{5}{|c|}{ Strength } & \multirow{2}{*}{$\begin{array}{l}\text { Modality } \\
\text { Exclusivity }\end{array}$} & \multirow[b]{2}{*}{ Characterization } \\
\hline & Visual & Haptic & Auditory & Olfactory & Gustatory & & \\
\hline Brunette & 4.8 & 0.0 & 0.1 & 0.0 & 0.0 & $98 \%$ & Unimodal visual (high exclusivity) \\
\hline Sparkly & 4.6 & 1.3 & 1.2 & 0.1 & 0.2 & $60 \%$ & Unimodal visual (moderate exclusivity) \\
\hline Jagged & 4.7 & 4.8 & 0.6 & 0.2 & 0.5 & $42 \%$ & Bimodal visual-haptic (moderate exclusivity) \\
\hline Rustling & 3.0 & 2.5 & 4.7 & 0.6 & 0.2 & $41 \%$ & $\begin{array}{l}\text { Multimodal auditory, moderately visual-haptic } \\
\text { (moderate exclusivity) }\end{array}$ \\
\hline Fresh & 4.0 & 2.4 & 1.0 & 4.5 & 4.1 & $22 \%$ & $\begin{array}{l}\text { Multimodal gustatory-olfactory-visual, } \\
\text { moderately haptic (low exclusivity) }\end{array}$ \\
\hline
\end{tabular}

apple: shiny) in a property verification task. If controlling modality strength and exclusivity eliminates cross-modal noise from the stimulus set (e.g., the auditory word rustling also has a significant visual presence [see Table 4], which could diminish the attentional cost of switching to the visual modality for shiny), we should find a lower degree of error in the resulting data set and a greater effect size for modality-switching costs.

\section{Method}

Participants. Twenty-five native speakers of English, with no reported reading or sensory disabilities, participated in the experiment for course credit. The participants were recruited via university e-mail lists and notice boards.

Materials. Forty strongly unimodal words were selected per modality and were attached to relevant objects (e.g., moth: speckled, keys: jingling). Two independent raters verified the appropriateness of all 200 attributions. We then formed pairs of object-property items for sequential presentation by randomly selecting an item from one modality (to be presented first) and pairing it with another, same- or different-modality item (the target). The pairing of each target item with its preceding modality was counterbalanced; for example, a visual item would be presented following another visual item (the same-modality condition), as well as following haptic, auditory, olfactory, and gustatory items (the different-modality conditions). Each participant saw every item, but in only one of these five possible pairs. Thus, every target item appeared in both sameand different-modality conditions. The strength of target items on the dominant modality was high $(M=4.52, S D=0.35)$, whereas strength on other modalities was low $(M=1.24, S D=1.35)$, resulting in moderate exclusivity $(M=0.44, S D=0.13)$.

A list of 300 object-property fillers was also created, 250 false and 50 true, to provide an overall balance of 50:50 true:false responses per participant. As in Pecher et al.'s (2003) Experiment 1, most of the false fillers were associated in Nelson, McEvoy, and Schreiber's (1998) word association norms (e.g.. oven: baked, coffin: dead) in order to ensure that the participants could not perform the task using simple word association strategies (Solomon \& Barsalou, 2004).

Procedure. The participants read instructions that asked them to press the button labeled "true" (the comma key) if the property was usually true of the concept but to press the button labeled "false" (the period key) if not. We used Pecher et al.'s (2003) example, carnation can be black, to highlight that, although carnations could theoretically be black, it would be highly unusual and should be judged as false. Each trial began with a fixation cross for $200 \mathrm{msec}$, followed by the item in the form object can be property, which stayed onscreen until the participant responded. The participants received immediate feedback if they responded incorrectly or too slowly (more than 2,000 msec), and each trial ended with a 200 -msec blank screen. A practice session of 24 items, half true and half false, preceded the main experiment. Critical pairs and fillers appeared in a random order, with a self-paced break every 100 trials.

\section{Results and Discussion}

One participant was excluded for having an accuracy rate of less than $70 \%$ on test items. Any target trials more than three standard deviations away from a participant's mean were removed as outliers ( $1.5 \%$ of the data). Only correct responses were analyzed, with comparable error rates across conditions (different $=7.1 \%$, same $=$ $6.9 \%)$.

People took longer to process object properties that were from a modality different from that on the previous trial $(M=1,117 \mathrm{msec}, S D=105.6)$ than those from the same modality $(M=1,083 \mathrm{msec}, S D=87.0)[t(23)=$ $2.81, p=.010]$. As was expected, the modality-switching cost (34 msec) replicated Pecher et al.'s (2003) findings. Since Pecher et al. did not report explicit effect sizes, we used G*Power (Faul, Erdfelder, Lang, \& Buchner, 2007) to estimate their switching cost effect size, using the reported means and standard errors for the same- and different-modality conditions (Experiment $1, \mathrm{SOA}=$ $0 \mathrm{msec}$ ), arriving at $d_{z}=0.192$. Similar analysis of our own data revealed a markedly larger effect size of $d_{z}=$ 0.513 , which confirms that using our normed words as stimuli in modality-specific tasks reduces noise in the data set. $^{2}$

\section{CONCLUSION}

We have presented modality exclusivity norms for a set of 423 object properties, including ratings of experiential strength on five perceptual modalities (visual, haptic, auditory, olfactory, and gustatory). Analysis shows that most properties are multimodal (i.e., perceived through multiple senses) rather than unimodal (i.e., perceived through one sense alone), with clustering of visual-haptic and olfactory-gustatory modalities. When researchers wish to investigate modality-specific issues in representation, our evaluation demonstrates that these norms can help produce cleaner stimulus sets that allow more subtle effects and interactions to emerge.

The nature of conceptual representation has enjoyed enhanced attention in recent years, since perceptual and conceptual processing has been seen as less of a dichotomy and more of a continuum. Increasing numbers of studies seek to increase our understanding of how conceptual thought may be grounded, such as by examining the representational roles of the sensory cortices or by rep- 
licating perceptual phenomena in conceptual tasks. Our hope is that the wider research community will find these norms useful in a broad range of experimental paradigms in which linguistic stimuli are used to investigate the relationship between perception and conception.

\section{AUTHOR NOTE}

This work was funded by Grant RES-000-22-2407 from the U.K. Economic and Social Research Council to the second author. Correspondence concerning this article should be addressed to L. Connell, School of Psychological Sciences, University of Manchester, Oxford Road, Manchester M13 9PL, England (e-mail: louise.connell@manchester.ac.uk).

\section{REFERENCES}

Amedi, A., von Kriegstein, K., van Atteveldt, N. M., Beauchamp, M. S., \& NAUMER, M. J. (2005). Functional imaging of human crossmodal identification and object recognition. Experimental Brain Research, 166, 559-571.

Balota, D. A., Yap, M. J., Cortese, M. J., Hutchison, K. A., Kessler, B., Loftis, B., ET AL. (2007). The English Lexicon Project. Behavior Research Methods, 39, 445-459.

Barsalou, L. W. (1999). Perceptual symbol systems. Behavioral \& Brain Sciences, 22, 577-660.

Barsalou, L. W. (2008). Grounded cognition. Annual Review of Psychology, 59, 617-645.

The British National Corpus, Version 2 (BNC WorLd) (2001). Distributed by Oxford University Computing Services on behalf of the BNC Consortium. Available at www.natcorp.ox.ac.uk.

Cimpian, A., \& Markman, E. M. (2008). Preschool children's use of cues to generic meaning. Cognition, 107, 19-53.

Connell, L. (2007). Representing object colour in language comprehension. Cognition, 102, 476-485.

ERnst, M. O., \& Bülthoff, H. H. (2004). Merging the senses into a robust percept. Trends in Cognitive Sciences, 8, 162-169.

Faul, F., Erdfelder, E., Lang, A.-G., \& Buchner, A. (2007). G*Power 3: A flexible statistical power analysis program for the social, behavioral, and biomedical sciences. Behavior Research Methods, 39, 175-191.

GlenberG, A. M., \& KaschaK, M. P. (2002). Grounding language in action. Psychonomic Bulletin \& Review, 9, 558-565.

Goldberg, R. F., Perfetti, C. A., \& Schneider, W. (2006). Perceptual knowledge retrieval activates sensory brain regions. Journal of $\mathrm{Neu}$ roscience, 26, 4917-4921.

González, J., Barros-Loscertales, A., Pulvermüller, F., MeSeguer, V., Sanjuán, A., Belloch, V., \& Ávila, C. (2006). Reading cinnamon activates olfactory brain regions. Neurolmage, 32, 906-912.

JoHnSON-LAIRD, P. N. (1983). Mental models: Towards a cognitive science of language, inference, and consciousness. Cambridge, MA Harvard University Press.

Kintsch, W. (2001). Predication. Cognitive Science, 25, 173-202.

LOCKE, J. (1975). An essay concerning human understanding (P. H. Nidditch, Ed.). Oxford: Oxford University Press, Clarendon Press (Original work published 1690)

MARQUES, J. F. (2006). Specialization and semantic organization: Evidence for multiple semantics linked to sensory modalities. Memory \& Cognition, 34, 60-67.

Martin, A. (2007). The representation of object concepts in the brain. Annual Review of Psychology, 58, 25-45.

McRae, K., Cree, G. S., Seidenberg, M. S., \& McNorgan, C. (2005). Semantic feature production norms for a large set of living and nonliving things. Behavior Research Methods, Instruments, \& Computers, 37, 547-559.

Medin, D. L., \& Schaffer, M. M. (1978). Context theory of classification learning. Psychological Review, 85, 207-238.

Nelson, D. L., McEvoy, C. L., \& Schreiber, T. A. (1998). The Uni- versity of South Florida word association, rhyme, and word fragment norms. Available at www.usf.edu/FreeAssociation.

Newman, S. D., Klatzky, R. L., Lederman, S. J., \& Just, M. A. (2005). Imagining material versus geometric properties of objects: An fMRI study. Cognitive Brain Research, 23, 235-246.

Osherson, D. N., \& SMITH, E. E. (1981). On the adequacy of prototype theory as a theory of concepts. Cognition, 9, 35-58.

Pecher, D., Zeelenberg, R., \& Barsalou, L. W. (2003). Verifying properties from different modalities for concepts produces switching costs. Psychological Science, 14, 119-124.

Pecher, D., \& ZwaAn, R. A. (EdS.) (2005). The grounding cognition: The role of perception and action in memory, language, and thinking. Cambridge: Cambridge University Press.

PulvermüLleR, F. (2005). Brain mechanisms linking language and action. Nature Reviews Neuroscience, 6, 576-582.

Rosch, E., \& Mervis, C. B. (1975). Family resemblances: Studies in the internal structure of categories. Cognitive Psychology, 7, 573-605.

Simmons, W. K., Ramjee, V., Beauchamp, M. S., McRae, K., MarTIN, A., \& BARSALOU, L. W. (2007). A common neural substrate for perceiving and knowing about color. Neuropsychologia, 45, 2802-2810.

Sloman, S., \& Malt, B. (2003). Artifacts are not ascribed essences, nor are they treated as belonging to kinds. Language \& Cognitive Processes, 18, 563-582.

Smith, L. B., \& SAmuelson, L. K. (2006). An attentional learning account of the shape bias: Reply to Cimpian and Markman (2005) and Booth, Waxman, and Huang (2005). Developmental Psychology, 42, 1339-1343.

Solomon, K. O., \& Barsalou, L. W. (2004). Perceptual simulation in property verification. Memory \& Cognition, 32, 244-259.

Spence, C., Nicholls, M. E. R., \& Driver, J. (2001). The cost of expecting events in the wrong sensory modality. Perception \& Psychophysics, 63, 330-336.

TuCKER, M. (2007). Slide Generator: A DirectX based Experiment Generator for Psychology (Version 2007.3.3). Available at www.psy plymouth.ac.uk/research/mtucker/SlideGenerator.htm.

Turatto, M., Benso, F., Galfano, G., \& Umiltà, C. (2002). Non-spatial attentional shifts between audition and vision. Journal of Experimental Psychology: Human Perception \& Performance, 28, 628-639.

Tyler, L. K., \& Moss, H. E. (2001). Towards a distributed account of conceptual knowledge. Trends in Cognitive Sciences, 5, 244-252.

Vinson, D. P., \& VigLIocco, G. (2008). Semantic feature production norms for a large set of objects and events. Behavior Research Methods, 40, 183-190.

Warrington, E. K., \& Shallice, T. E. (1984). Category specific semantic impairments. Brain, 107, 829-853.

WILSON, M. D. (1988). MRC Psycholinguistic Database: Machineusable dictionary, version 2.00. Behavior Research Methods, Instruments, \& Computers, 20, 6-10.

ZwaAn, R. (2004). The immersed experiencer: Toward an embodied theory of language comprehension. Psychology of Learning \& Motivation, 44, 35-62.

\section{NOTES}

1. The order of modalities in the rating scales was fixed in this order to minimize participant errors when moving between items. There is no evidence in the literature to suggest that switching from one modality to another causes a qualitative change in the resulting representation (making, e.g., a property like murky seem less visual after a participant has thought about touching and hearing it).

2 . The same correlation between conditions $(r=.78$, taken from our own study) was used in the calculation of both effect sizes.

\section{SUPPLEMENTAL MATERIAL}

The modality exclusivity norms may be downloaded as supplemental material for this article from brm.psychonomic-journals.org/content/ supplemental. 
APPENDIX

Variables Included in the Norms File

\begin{tabular}{|c|c|}
\hline Variable & Definition \\
\hline Item\# & Number of property word in alphabetical order (1-423) \\
\hline Property & Word that describes target property \\
\hline Familiarity & $\begin{array}{l}\text { Proportion of participants that were familiar with the meaning of the property } \\
\text { word }(0 \%-100 \%)\end{array}$ \\
\hline VisualStrengthMean & Mean rating $(0-5)$ of how strongly property is experienced by seeing \\
\hline HapticStrengthMean & Mean rating $(0-5)$ of how strongly property is experienced by feeling through touch \\
\hline AuditoryStrengthMean & Mean rating $(0-5)$ of how strongly property is experienced by hearing \\
\hline OlfactoryStrengthMean & Mean rating $(0-5)$ of how strongly property is experienced by smelling \\
\hline GustatoryStrengthMean & Mean rating $(0-5)$ of how strongly property is experienced by tasting \\
\hline VisualStrengthSD & Standard deviation of visual strength ratings \\
\hline HapticStrengthSD & Standard deviation of haptic strength ratings \\
\hline AuditoryStrengthSD & Standard deviation of auditory strength ratings \\
\hline OlfactoryStrengthSD & Standard deviation of olfactory strength ratings \\
\hline GustatoryStrengthSD & Standard deviation of gustatory strength ratings \\
\hline DominantModality & $\begin{array}{l}\text { Modality through which property is experienced most strongly (i.e., with highest } \\
\text { strength rating) }\end{array}$ \\
\hline ModalityExclusivity & $\begin{array}{l}\text { Extent to which property is experienced though a single perceptual modality }(0 \%- \\
100 \%) \text {, calculated as range of strength values divided by their sum }\end{array}$ \\
\hline BNCFrequency & Frequency per million words in the British National Corpus (2001) \\
\hline BNCLogFrequency & $\begin{array}{l}\text { Natural logarithm of BNC frequency (one unit was added to the BNC frequency } \\
\text { prior to transform to allow for words of zero frequency) }\end{array}$ \\
\hline OrthographicLength & Number of letters in property word \\
\hline
\end{tabular}

(Manuscript received September 17, 2008; revision accepted for publication December 2, 2008.) 